\title{
Probing near-horizon fluctuations with black hole binary mergers
}

\author{
Steven L. Liebling, ${ }^{a}$ Matthew Lippert ${ }^{b}$ and Michael Kavic ${ }^{b}$ \\ ${ }^{a}$ Department of Physics, Long Island University, \\ Brookville, New York 11548, U.S.A. \\ ${ }^{b}$ Department of Physics, Long Island University, \\ Brooklyn, New York 11201, U.S.A. \\ E-mail: steve.liebling@liu.edu, matthew.lippert@liu.edu, \\ michael.kavic@liu.edu
}

ABSTRACT: The strong version of the nonviolent nonlocality proposal of Giddings predicts "strong but soft" quantum metric fluctuations near black hole horizons in an attempt to resolve the information paradox. To study observable signatures of this proposal, we numerically solve Einstein's equations modified by these fluctuations and analyze the gravitational wave signal from the inspiral and merger of two black holes. In a model of evolution for such fluctuations, we show that they lead to significant deviations in the observed waveform, even when the black holes are still well separated, and could potentially be observed by aLIGO.

KEYwords: Black Holes, Models of Quantum Gravity

ARXIV EPRINT: 1707.02299 


\section{Contents}

1 Introduction 1

2 Numerical system 3

3 Results 4

$\begin{array}{lll}4 & \text { Observational prospects } & 6\end{array}$

5 Discussion $\quad 8$

\section{Introduction}

The groundbreaking observations of binary black hole collisions by Advanced LIGO (aLIGO) have ushered in a new era of gravitational wave astronomy [1-3]. The gravitational wave signal emitted from the inspiral, coalescence, and ringdown of two black holes permits direct observation of extreme physics in the near-horizon region. Even the few observations to date contain a wealth of information which can be used to test proposed modifications to the conventional general relativistic picture of a black hole [4].

The semi-classical description of black hole collapse and evaporation leads to the information paradox, whose resolution has motivated proposals for radically new physics (see [5] and [6] for two recent reviews). If information is not irrevocably lost but somehow emerges from behind the horizon, the traditional description in terms of local quantum field theory on a semi-classical geometry must be modified, and not just by small, subtle effects [7-9]. A number of such drastic resolutions have been suggested; for example, the locally harmless horizon could be replaced with a hard and deadly firewall [8] or the entire black hole geometry might be replaced by a non-geometric fuzzball [9].

The nonviolent nonlocality proposal [10-15] (also [16]), by contrast, does not eliminate the event horizon. Local low-energy quantum field theory outside the horizon is supplemented by nonlocal couplings with degrees of freedom inside the black hole, leading to soft quantum metric fluctuations whose wavelengths are not set by the Planck scale but instead by the much larger Schwarzschild radius. According the strong version of the proposal, these are unit-size fluctuations in order to allow information to escape at the necessary rate.

These types of significant modifications to the standard black hole picture might be expected to have observable consequences. Much of the recent focus has been on the prospects for imaging the supermassive black hole Sagittarius A* using the Event Horizon Telescope $[13,17]$. High-precision timing measurements from a suitable pulsar-black hole binary could also detect large-amplitude quantum fluctuations [18].

However, the gravitational wave signal from a black hole binary merger is currently the most promising venue in which to hunt for signs of new physics and to constrain deviations 
from general relativity (GR). Several recent attempts have been made to model the gravitational wave predictions of various alternative theories (e.g. [19, 20]). One approach [21-24] is to replace the black hole with an object nearly as compact, a so-called "exotic compact object" and explore any observational differences due solely to the removal of the horizon. In [25], the horizon was replaced with a reflecting boundary as a proxy for a firewall and faint echoes of the original signal were reported in the late stages of the ringdown. ${ }^{1}$

It should be noted that the requirement that the quantum fluctuations have order-one amplitudes has recently been questioned [15]. In this weaker version of the proposal, the fluctuations of the geometry may be exponentially suppressed, rendering any astrophysical signals completely unobservable.

In this paper, we focus on the original, stronger nonviolent nonlocality proposal and analyze the resulting modifications to the binary black hole merger signal. We numerically evolve the Einstein equations for two equal-mass black holes, colliding them both head-on and in a quasi-circular inspiral, implementing the quantum effects as Schwarzschild-scale metric fluctuations, supported within roughly a Schwarzschild radius of the horizon. We vary the both the amplitude and frequency of the metric fluctuations.

In anticipating the observational effect of the nonviolent nonlocality scenario, we should emphasize (as noted by [14]) that the proposed quantum fluctuations occur near the horizon, and so the metric well outside the black hole might be expected to resemble that of a standard black hole without such fluctuations. Similarly, the spacetime outside a neutron star is the same as that of a black hole of equal mass. Of course, the neutron star lacks the black hole's horizon, but this difference is relatively unimportant in a well separated binary system. The distinction between black hole and neutron star binaries only becomes apparent when the constituents are sufficiently close that the structure of the objects begins to affect the dynamics. ${ }^{2}$ Because the modifications in the nonviolent nonlocality scenario are confined to the near-horizon region, this analogy with a neutron start suggests that the nonviolent nonlocality proposal would also have minimal dynamical implications until just before the merger. However, the numerical results we present below suggest otherwise.

We find that the fluctuations yield significant deviations from the baseline GR signal. Within the frequency band where aLIGO is sensitive, we calculate the overlap between the merger waveform derived from GR and the one arising when the metric fluctuations are present. Our results imply that the strong version of the nonviolent nonlocality proposal produces potentially observable effects for nominal choices of parameters. However, deviations due to the fluctuations could conceivably be mimicked by changes in other parameters, such as black hole masses or spins. Extensive further numerical studies would be needed to definitively rule out this possibility.

Contrary to the expectations of [14], which argued that the effects of quantum fluctuations would appear only in the very late inspiral and merger process, we find these effects are already present during the early inspiral phase. This suggests that the impact of the fluctuations radiates beyond their near-horizon region of support in a manner very different from a firewall or other exotic compact object.

\footnotetext{
${ }^{1}$ These claims have been controversial; see [26-29].

${ }^{2}$ See Damour's "effacement theorem" [30].
} 


\section{Numerical system}

We begin with an existing code which makes use of the BSSN formulation [31, 32] to implement the Einstein equations. This code has been tested without the fluctuations described here extensively and used to study a number of different phenomena [33-35]. In this approach, the spacetime metric is written as

$$
d s^{2}=-\alpha^{2} d t^{2}+\gamma_{i j}\left(d x^{i}+\beta^{i} d t\right)\left(d x^{j}+\beta^{j} d t\right),
$$

with $\alpha$ the lapse function and $\beta^{i}$ the shift vector. Specifically, we express the Einstein equations in terms of the BSSN-NOK formalism [31, 32, 36, 37]. In this formulation, the metric on spatial hypersurfaces $\gamma_{i j}$ is expressed in terms of a conformal factor $\chi$ and a conformally flat metric $\tilde{\gamma}_{i j}$ as

$$
\gamma_{i j}=\frac{1}{\chi} \tilde{\gamma}_{i j}, \quad \chi=\left(\operatorname{det} \gamma_{i j}\right)^{-1 / 3}
$$

such that $\operatorname{det} \tilde{\gamma}_{i j}=1$.

The initial data consists of the superposition of two equal-mass black holes (for details, see [38]). ${ }^{3}$ We consider two types of initial momenta: the black holes boosted directly toward each other leading to a head-on collision, and the black holes given opposite transverse boosts to achieve an approximately quasi-circular orbit. We work in units of the total mass of the system $M$. Each black hole, therefore, has mass roughly $M / 2$.

We then modify the code to incorporate the metric fluctuations. The nonviolent nonlocality scenario proposes a nonlocal coupling between operators inside the black hole and the exterior stress tensor [11] which, when integrated out, acts as a quantum source for the metric. In our code, this effect is represented as a fluctuating classical source perturbing the metric. Because the source is supported in the near-horizon region, the location of the source must constantly be updated to track the moving black holes.

We take a simple, somewhat ad hoc implementation which captures the essential physics. At each step in the numerical integration, we modify by hand the time derivatives of the diagonal components of the metric ${ }^{4}$

$$
\begin{aligned}
\dot{\alpha} & \rightarrow \dot{\alpha}(1+H) \\
\dot{\tilde{\gamma}}_{i i} & \rightarrow \dot{\tilde{\gamma}}_{i i}(1-H) .
\end{aligned}
$$

For the perturbation $H$, we postulate the following form

$$
H=A \exp ^{-\left(\vec{r}-\vec{r}_{1 \mid 2}\right)^{2} / R^{2}} \sin (\Omega t),
$$

where $A$ is the amplitude and $\Omega$ is the angular frequency. We assume a Gaussian radial profile of width $R$ centered on the locations of the two black holes. The coordinate location

\footnotetext{
${ }^{3}$ Note that these black holes will quickly evolve consistent with the puncture method to have a "trumpet" topology and thus differ from Schwarzschild black holes; for details, see [38].

${ }^{4}$ The $\pm H$ helps avoid signature changes of the metric as explained in section III of ref. [17].
} 
of the nearer black hole is denoted by $\vec{r}_{1 \mid 2}$, and we track these locations by monitoring the minimum values of $\chi$.

The metric perturbation $H$ in the nonviolent nonlocality proposal has an order-one amplitude, has support within a region roughly a Schwarzschild radius from the horizon, and has a frequency on the order of the inverse light-crossing time of the black hole. ${ }^{5}$ As the exact values of these parameters are not specified by the nonviolent nonlocality model, we will scan over a range such that: $A \lesssim 1, R / M \approx 1$, and $M \Omega \approx 1$.

We adopt a Courant parameter of $\lambda=0.25$ using a self-shadow adaptive mesh method with seven levels of dynamic refinement achieving a finest resolution of $0.04 M$.

To extract physical information, we monitor the gravitational Newman-Penrose radiative scalar $\Psi_{4}$ on spherical shells located in the wave zone (typically with radius $80 M$ ). This quantity is computed by contracting the Weyl tensor with a suitably defined null tetrad,

$$
\Psi_{4}=C_{a b c d} n^{a} \bar{m}^{b} n^{c} \bar{m}^{d}
$$

and accounts for the energy carried off by outgoing waves at infinity.

The strain $h(t)$, which is directly observed by aLIGO, is related to the gravitational scalar by

$$
\frac{d^{2}}{d t^{2}} h=\Psi_{4}
$$

\section{Results}

Despite the ad hoc nature of the modifications, the evolution is well behaved. We compute the $L^{2}$-norms of the Hamiltonian constraint and the momentum constraints. The residuals of the modified and GR evolutions are comparable, suggesting that our numerical scheme to modify gravity remains reasonable throughout the evolution.

Second, we display in figure 1 the gravitational wave signal due to a head-on collision with quantum fluctuations, computed at two different resolutions, along with the unmodified GR computation. It seems unlikely, although not impossible, the that added fluctuations (2.3) can originate from a discretized set of continuum differential equations. And, if we are not solving a system of differential equations with a unique, continuum solution, true convergence is not expected.

However, if the results were highly dependent on resolution, it would be difficult to make predictions. As indicated in the figure the results are, to a good approximation, independent of resolution.

Head-on collisions provide only a very short time window for quantum fluctuations to have an effect. However, they do provide a measure of the quasi-normal modes of an out-of-equilibrium black hole, namely the remnant black hole as it settles to a quiescent state. As shown in figure 1, compared with the GR computation, the quantum fluctuations do result in differences in the brief, pre-merger signal, but the ringdown is largely the same for all the evolutions.

\footnotetext{
${ }^{5}$ We work in units where Newton's constant $G=1$. For a black hole of mass $M / 2$, the Schwarzschild radius is $M$.
} 


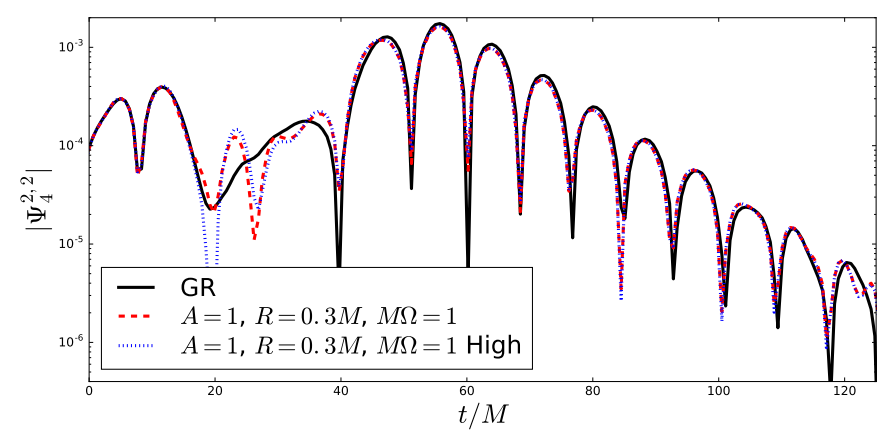

Figure 1. Signals from a head-on collision for two resolutions. Although not fully converged because there is no continuum solution, the high- and low-resolution results are very similar, suggesting the scheme is reasonably well-behaved.

Note that the quantum fluctuations couple only to the dynamics, in that they are introduced as multiplicative factors to the time derivatives of the metric (2.3). Hence, after the merger, when the remnant is settling down, the time derivatives become small and thus so do these modifications. One could alternatively consider some modification that coupled directly to the metric instead of its time derivatives.

The quasi-circular inspiral is more sensitive to quantum fluctuations than the headon collision both because it takes longer for the merger to occur and also because the breaking of axial symmetry allows more complex motion. It was previously observed with the Einstein-Maxwell-dilaton system studied in [19] that orbital mergers were much more sensitive probes of deviations from GR than head-on collisions, and we find this to be true here as well.

In particular, quantum fluctuations in the neighborhood around the black holes introduce eccentricity in the black hole orbits and produce high frequency features in the gravitational wave signatures.

We present results from orbital coalescences in figures 2 and 3 , showing a range of fluctuation amplitudes $A$ and frequencies $\Omega$, respectively. The three panels in each figure display the (dominant) $l=2=m$ component of the radiative scalar $\Psi_{4}^{2,2}$ in the top panel, the real component of its corresponding wave strain $h$ in the middle panel, and the angular frequency $\omega$ of the binary as a function of time. These figures illustrate significant differences between the nonviolent nonlocality model and the GR prediction.

In order to visualize the dynamical effects of these modifications, we subtract the GR solution as a function of time from an evolution with fluctuations. Figure 4 shows this difference for the conformal factor $\chi$ at four different times and illustrates the effect of the fluctuations. Qualitatively, it indicates that the deviation from GR manifests dynamically as a coherent oscillating wave. During the inspiral, this oscillation appears roughly quadrupolar. In contrast, after the merger, the amplitude decreases significantly and the oscillation is primarily monopolar. 

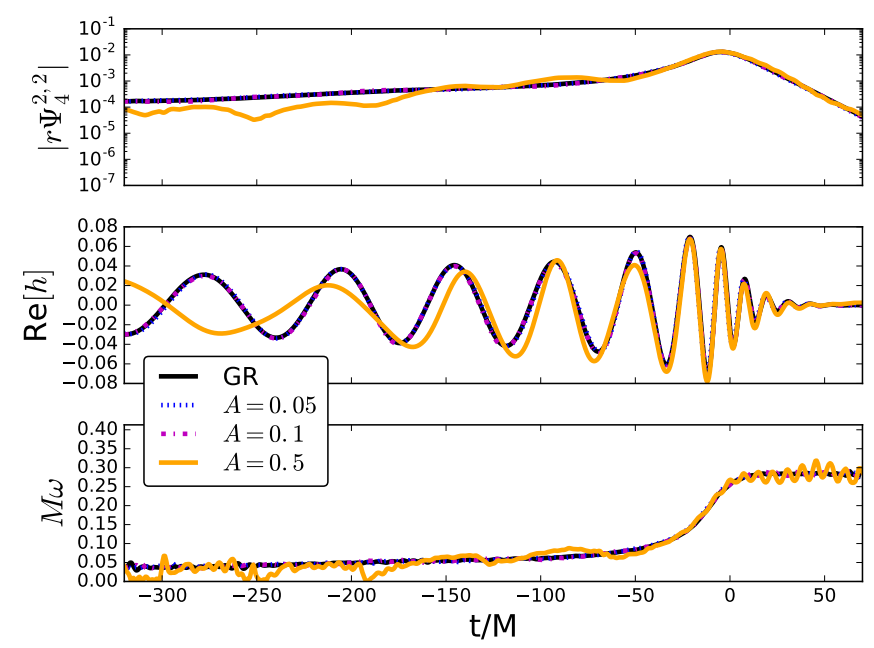

Figure 2. Results for the inspiral and coalescence of two, equal-mass black holes, for varying amplitudes $A$ of the quantum fluctuations. These runs have $M \Omega=0.1$ and $R_{0}=0.3 M$. The times and overall phases of the nonviolent nonlocality waveforms have been shifted to maximize the overlap with the GR waveform. Top: the magnitude of the gravitational wave scalar $\left|r \Psi_{4}^{2,2}\right|$. Middle: the real component of the strain $h$. Bottom: the angular frequency $\omega$ of the binary.
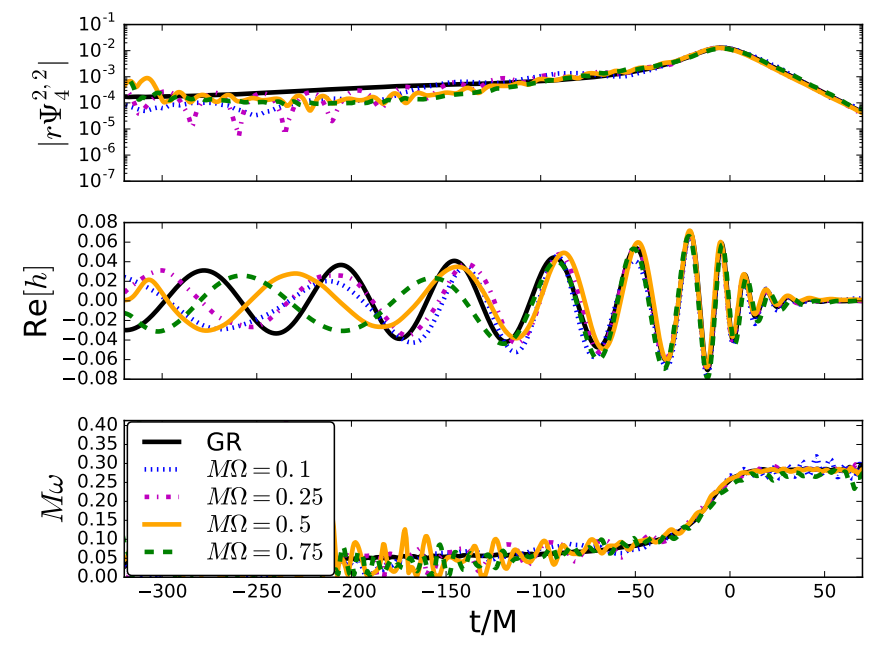

Figure 3. Results of varying the fluctuation frequency $\Omega$ for the inspiral and coalescence of two, equal-mass black holes. These runs have $A=0.5 M$ and $R_{0}=0.3 M$. The same quantities as shown in figure 2 are presented here, and again the times and overall phases of the nonviolent nonlocality waveforms have been shifted to maximize the overlap with the GR waveform.

\section{Observational prospects}

We have shown qualitatively in figures 2 and 3 that quantum fluctuations modify the gravitational wave signal. However, we need to quantify the extent to which these modified waveforms can be distinguished from the standard GR waveform by a gravitational wave detector such as aLIGO. 


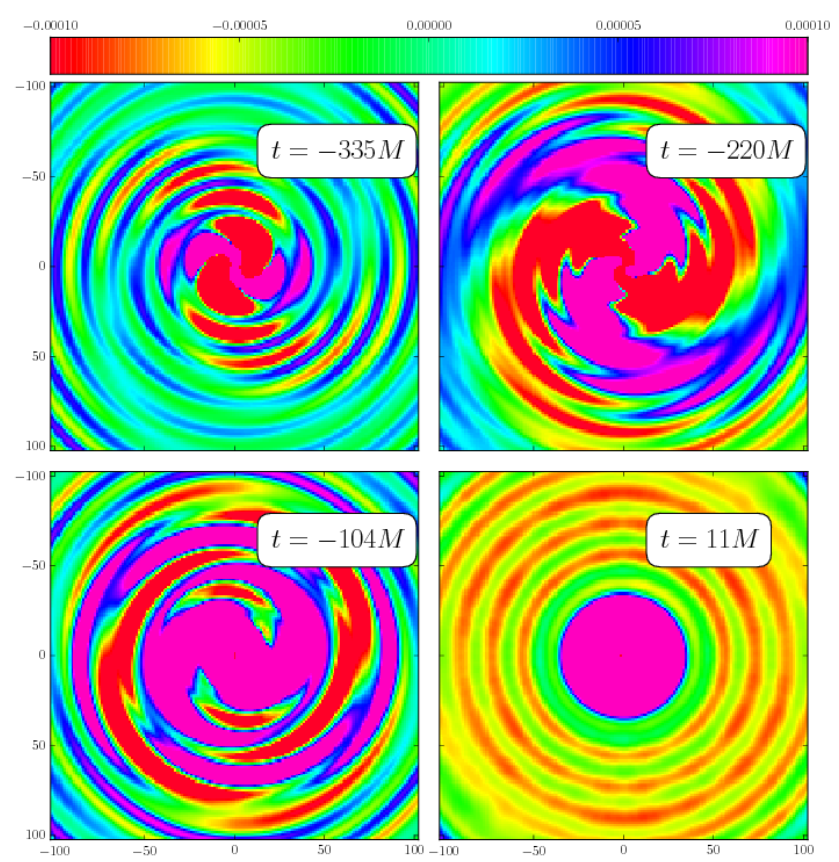

Figure 4. Difference in the conformal factor $\chi$ on the orbital plane between an inspiral with $A=0.5, R_{0}=0.3 M$, and $M \Omega=0.5$ and the run without quantum fluctuations. The black hole binary rotates clockwise and has merged by the final frame shown. The colormap is the same for all frames and has been chosen to emphasize the far zone. Note the change in character of the far-zone behavior after the merger, where the difference radiates symmetrically.

In order to assess the observability of the deviation, we compute the overlap between the perturbed waveforms and the standard GR waveform using methods from [39-41]. For two gravitational wave signals with strains $h_{1}(t)$ and $h_{2}(t)$, the overlap is given by the normalized inner product

$$
\mathcal{O}(1,2)=\max \frac{\langle 1 \mid 2\rangle}{\sqrt{\langle 1 \mid 1\rangle\langle 2 \mid 2\rangle}}
$$

maximized over time and phase shifts. In order to take into account the sensitivity of aLIGO over its bandwidth, we employ a frequency-domain, noise-weighted, inner product

$$
\langle 1 \mid 2\rangle=\operatorname{Re} \int_{-\infty}^{\infty} \frac{\tilde{h}_{1}(\omega) \tilde{h}_{2}^{*}(\omega)}{S(\omega)},
$$

where $\tilde{h}_{1}(\omega)$ and $\tilde{h}_{2}(\omega)$ are the Fourier transforms of $h_{1}(t)$ and $h_{2}(t)$. The integrand is weighted by the noise profile of aLIGO $S(\omega)$, available at $[42] .{ }^{6}$

Because gravitational wave signals scale with the total mass $M$ of the binary pair, we must specify $M$ in order to determine the overlap across the sensitivity band of aLIGO. Table 1 presents the overlap $\mathcal{O}$ for all of the choices of fluctuation amplitude $A$ and frequency $M \Omega$ shown in figures 2 and 3, each for several values of $M$.

\footnotetext{
${ }^{6}$ The LIGO noise profile ranges from $9 \mathrm{~Hz}$ to $8192 \mathrm{~Hz}$, so in practice the integral in (4.2) is cut off at those limits.
} 


\begin{tabular}{|ll|ccc|}
\hline & & \multicolumn{3}{|c|}{ Overlap $\mathcal{O}$ for } \\
$A$ & $\mathrm{M} \Omega$ & $M=6 M_{\odot}$ & $M=20 M_{\odot}$ & $M=60 M_{\odot}$ \\
\hline 0.05 & 0.1 & 0.997 & 0.996 & 0.996 \\
0.1 & 0.1 & 0.999 & 0.999 & 0.999 \\
0.5 & 0.1 & 0.694 & 0.764 & 0.827 \\
0.5 & 0.25 & 0.757 & 0.802 & 0.840 \\
0.5 & 0.5 & 0.492 & 0.599 & 0.692 \\
0.5 & 0.75 & 0.517 & 0.616 & 0.702 \\
\hline
\end{tabular}

Table 1. The overlap $\mathcal{O}$ between the GR waveform and the perturbed waveforms with amplitudes $A$ and frequencies $M \Omega$, computed using eq. (4.1).

As would be expected, fluctuations with larger amplitude have smaller overlap with the GR waveform. Higher frequency fluctuations (at a fixed $M$ ) also leads to smaller overlap.

Varying the total mass has a notable effect on the overlap. aLIGO is most sensitive in a frequency band around $245 \mathrm{~Hz}$, and for $M=60 M_{\odot}$, very roughly the total mass of GW150914 $\left(65 M_{\odot}\right)$ and GW170104 $\left(50 M_{\odot}\right)$, the power spectrum of the signal is peaked at frequencies around $50 \mathrm{~Hz}$. Decreasing $M$ shifts the gravitational wave signal toward higher frequency and better sensitivity. Consequently, overlaps are generally lower for smaller masses.

With these overlaps computed, we need to establish the threshold at which aLIGO is able to distinguish the two waveforms. Ref. [41] argues that a mismatch $\mathcal{M}=1-\mathcal{O}$ exceeding the inverse square of the signal-to-noise ratio will be observable. The signal to noise ratio for GW150914 was 24, implying that waveforms with overlap less than 0.998 would be distinguishable. Here, we will adopt a more conservative standard of $\mathcal{O} \lesssim 0.97$ for distinguishability, a typical minimum overlap for neighboring points in template banks [43-45], for which a signal to noise ratio of only 5.8 would be required.

Using this standard, table 1 indicates that nominal amplitudes and frequencies suggested by Giddings could be constrained by observations. Several binary mergers have so far been detected with no observable deviation from the standard GR waveform. This implies, in principle, these aLIGO observations could set limits on the parameter space of the nonviolent nonlocality model. However, establishing such a bound will require addressing several challenges, which we outline below.

\section{Discussion}

Our results indicate that the near-horizon fluctuations predicted by the strong nonviolent nonlocality proposal may significantly affect the orbital dynamics of inspiraling black hole binaries. Unlike the comparison between the geometry of a neutron star and that of a black hole of identical mass, these modifications to GR are radiative and impact the gravitational wave signal more when the black holes are well separated than just before they merge. And, we have furthermore argued that aLIGO can, in principle, observe these effects. We should note, however, a number of important caveats. 
It is possible that the modifications to the gravitational wave signal resulting from the quantum fluctuations could alternatively be produced by changing other parameters, such as the black hole masses, spins, or separation. In this case, even if the fluctuations did exist, the observed signal would be consistent with unmodified GR describing the inspiral of some alternate binary system. Conclusively ruling out such possible degeneracies would require a greatly extended overlap analysis across a range of parameters to determine the true distinguishability of the nonviolent nonlocality waveforms. Such extensive numerical computations are beyond the scope of this work; our goal is a proof of principle that quantum fluctuations yield observable gravitational wave signals.

The nonviolent nonlocality model proposes that the black hole solution is modified by quantum metric fluctuations but does not provide a consistent dynamical mechanism to produce them, such as a modification to Einstein's equation. As a consequence, the procedure by which the quantum fluctuations are numerically implemented is necessarily ad hoc, in that no continuum limit is being solved. As a result, alternative implementations are possible which might predict different or more subtle effects. For example, one could perturb the GR spacetime directly instead of the time derivatives.

Another alternative implementation could include an ensemble of fluctuations with different frequencies, which might yield more incoherent quantum effects. In particular, such a scheme might avoid any peculiarities of a coherent, single-frequency fluctuation interacting with the orbiting system. We have implemented such an ensemble with random frequencies chosen from a Gaussian distribution about a chosen central value and amplitudes chosen such that the "energies" $A_{i}^{2} \Omega_{i}^{2}$ of the fluctuations are also distributed in a Gaussian manner about a central energy. Furthermore, the amplitudes are rescaled so that the sum of the fluctuation energies matches the energy of a single mode. One might guess that these modes might interfere in such a way that their effects would tend to cancel out. However, early results show a significant effect, so much so that the binary components are no longer bound and immediately fly apart. Similar behavior was observed for amplitudes larger than those presented here.

Due to the lack of a continuum limit, numerical effects are not well understood. Although the behavior studied here does not appear sensitive to the resolution, moving away from the rigorous standard of convergence and consistency leaves room for uncertainty.

Despite these caveats, we argue that the method described here, even if it falls short of solving a fully nonlinear, consistent set of differential equations, represents a significant step toward a quantitative, observational test of the strong nonviolent nonlocality model. In this regard, our results suggest that such near-horizon fluctuations could be constrained by gravitational wave observations of binary black hole mergers.

\section{Acknowledgments}

We thank Peter Shawhan for both helpful discussions and comments on the manuscript. It is also a pleasure to thank Steve Giddings, Luis Lehner, Vassilios Mewes, Bob Wald, and Nico Yunes for helpfulcomments and discussions. This work was supported by the NSF under grants PHY-1308621 \& PHY-1607291 (LIU) and NASA's ATP program through grant NNX13AH01G. Computations were performed at XSEDE. 
Open Access. This article is distributed under the terms of the Creative Commons Attribution License (CC-BY 4.0), which permits any use, distribution and reproduction in any medium, provided the original author(s) and source are credited.

\section{References}

[1] Virgo, LIGO Scientific collaborations, B.P. Abbott et al., Observation of Gravitational Waves from a Binary Black Hole Merger, Phys. Rev. Lett. 116 (2016) 061102 [arXiv: 1602.03837] [INSPIRE].

[2] Virgo, LigO Scientific collaborations, B.P. Abbott et al., GW151226: Observation of Gravitational Waves from a 22-Solar-Mass Binary Black Hole Coalescence, Phys. Rev. Lett. 116 (2016) 241103 [arXiv:1606.04855] [INSPIRE].

[3] VIRGO, LIGO Scientific collaborations, B.P. Abbott et al., GW170104: Observation of a 50-Solar-Mass Binary Black Hole Coalescence at Redshift 0.2, Phys. Rev. Lett. 118 (2017) 221101 [arXiv: 1706.01812] [INSPIRE].

[4] Virgo, Ligo Scientific collaborations, B.P. Abbott et al., Tests of general relativity with GW150914, Phys. Rev. Lett. 116 (2016) 221101 [arXiv: 1602.03841] [INSPIRE].

[5] W.G. Unruh and R.M. Wald, Information Loss, Rept. Prog. Phys. 80 (2017) 092002 [arXiv: 1703.02140] [INSPIRE].

[6] D. Marolf, The Black Hole information problem: past, present and future, Rept. Prog. Phys. 80 (2017) 092001 [arXiv: 1703.02143] [INSPIRE].

[7] D.N. Page, Information in black hole radiation, Phys. Rev. Lett. 71 (1993) 3743 [hep-th/9306083] [INSPIRE].

[8] A. Almheiri, D. Marolf, J. Polchinski and J. Sully, Black Holes: Complementarity or Firewalls?, JHEP 02 (2013) 062 [arXiv:1207.3123] [INSPIRE].

[9] S.D. Mathur, The Fuzzball proposal for black holes: An Elementary review, Fortsch. Phys. 53 (2005) 793 [hep-th/0502050] [inSPIRE].

[10] S.B. Giddings, Models for unitary black hole disintegration, Phys. Rev. D 85 (2012) 044038 [arXiv: 1108.2015] [INSPIRE].

[11] S.B. Giddings, Nonviolent nonlocality, Phys. Rev. D 88 (2013) 064023 [arXiv:1211.7070] [INSPIRE].

[12] S.B. Giddings, Modulated Hawking radiation and a nonviolent channel for information release, Phys. Lett. B 738 (2014) 92 [arXiv:1401.5804] [InSPIRE].

[13] S.B. Giddings, Possible observational windows for quantum effects from black holes, Phys. Rev. D 90 (2014) 124033 [arXiv:1406.7001] [INSPIRE].

[14] S.B. Giddings, Gravitational wave tests of quantum modifications to black hole structure with post-GW150914 update, Class. Quant. Grav. 33 (2016) 235010 [arXiv:1602.03622] [INSPIRE].

[15] S.B. Giddings, Nonviolent unitarization: basic postulates to soft quantum structure of black holes, JHEP 12 (2017) 047 [arXiv: 1701.08765] [INSPIRE].

[16] A.Y. Yosifov and L.G. Filipov, Entropic Entanglement: Information Prison Break, Adv. High Energy Phys. 2017 (2017) 8621513 [arXiv:1707.01768] [INSPIRE]. 
[17] S.B. Giddings and D. Psaltis, Event Horizon Telescope Observations as Probes for Quantum Structure of Astrophysical Black Holes, arXiv: 1606.07814 [INSPIRE].

[18] J. Estes, M. Kavic, M. Lippert and J.H. Simonetti, Shining Light on Quantum Gravity with Pulsar-Black hole Binaries, Astrophys. J. 837 (2017) 87 [arXiv: 1607.00018] [INSPIRE].

[19] E.W. Hirschmann, L. Lehner, S.L. Liebling and C. Palenzuela, Black Hole Dynamics in Einstein-Maxwell-Dilaton Theory, arXiv:1706.09875 [INSPIRE].

[20] M. Okounkova, L.C. Stein, M.A. Scheel and D.A. Hemberger, Numerical binary black hole mergers in dynamical Chern-Simons gravity: Scalar field, Phys. Rev. D 96 (2017) 044020 [arXiv: 1705.07924] [INSPIRE].

[21] C. Barcelo, R. Carballo-Rubio, L.J. Garay and G. Jannes, The lifetime problem of evaporating black holes: mutiny or resignation, Class. Quant. Grav. 32 (2015) 035012 [arXiv: 1409.1501] [INSPIRE].

[22] V. Cardoso, S. Hopper, C.F.B. Macedo, C. Palenzuela and P. Pani, Gravitational-wave signatures of exotic compact objects and of quantum corrections at the horizon scale, Phys. Rev. D 94 (2016) 084031 [arXiv:1608.08637] [INSPIRE].

[23] E. Maggio, P. Pani and V. Ferrari, Exotic Compact Objects and How to Quench their Ergoregion Instability, Phys. Rev. D 96 (2017) 104047 [arXiv: 1703. 03696] [INSPIRE].

[24] Z. Mark, A. Zimmerman, S.M. Du and Y. Chen, A recipe for echoes from exotic compact objects, Phys. Rev. D 96 (2017) 084002 [arXiv:1706.06155] [inSPIRE].

[25] J. Abedi, H. Dykaar and N. Afshordi, Echoes from the Abyss: Tentative evidence for Planck-scale structure at black hole horizons, Phys. Rev. D 96 (2017) 082004 [arXiv: 1612.00266] [INSPIRE].

[26] G. Ashton et al., Comments on: "Echoes from the abyss: Evidence for Planck-scale structure at black hole horizons", arXiv:1612.05625 [INSPIRE].

[27] J. Abedi, H. Dykaar and N. Afshordi, Echoes from the Abyss: The Holiday Edition!, arXiv: 1701.03485 [INSPIRE].

[28] C. Barceló, R. Carballo-Rubio and L.J. Garay, Gravitational wave echoes from macroscopic quantum gravity effects, JHEP 05 (2017) 054 [arXiv: 1701.09156] [INSPIRE].

[29] R.H. Price and G. Khanna, Gravitational wave sources: reflections and echoes, Class. Quant. Grav. 34 (2017) 225005 [arXiv:1702.04833] [INSPIRE].

[30] T. Damour, The problem of motion in newtonian and einsteinian gravity, in Three Hundred Years of Gravitation, S. Hawking and W. Israel eds., Cambridge University Press, Cambridge, New York (1987), pp. 128-198 [INSPIRE].

[31] M. Shibata and T. Nakamura, Evolution of three-dimensional gravitational waves: Harmonic slicing case, Phys. Rev. D 52 (1995) 5428 [INSPIRE].

[32] T.W. Baumgarte and S.L. Shapiro, On the numerical integration of Einstein's field equations, Phys. Rev. D 59 (1999) 024007 [gr-qc/9810065] [INSPIRE].

[33] C. Palenzuela, M. Anderson, L. Lehner, S.L. Liebling and D. Neilsen, Stirring, not shaking: binary black holes' effects on electromagnetic fields, Phys. Rev. Lett. 103 (2009) 081101 [arXiv: 0905.1121] [INSPIRE].

[34] D. Neilsen et al., Boosting jet power in black hole spacetimes, Proc. Nat. Acad. Sci. 108 (2011) 12641 [arXiv:1012.5661] [InSPIRE]. 
[35] S.L. Liebling and C. Palenzuela, Electromagnetic Luminosity of the Coalescence of Charged Black Hole Binaries, Phys. Rev. D 94 (2016) 064046 [arXiv:1607.02140] [InSPIRE].

[36] T. Nakamura, K. Oohara and Y. Kojima, General Relativistic Collapse to Black Holes and Gravitational Waves from Black Holes, Prog. Theor. Phys. Suppl. 90 (1987) 1 [INSPIRE].

[37] M. Campanelli, C.O. Lousto, P. Marronetti and Y. Zlochower, Accurate evolutions of orbiting black-hole binaries without excision, Phys. Rev. Lett. 96 (2006) 111101 [gr-qc/0511048] [INSPIRE].

[38] M. Hannam, S. Husa and N.O. Murchadha, Bowen-York trumpet data and black-hole simulations, Phys. Rev. D 80 (2009) 124007 [arXiv:0908.1063] [INSPIRE].

[39] C. Cutler and E.E. Flanagan, Gravitational waves from merging compact binaries: How accurately can one extract the binary's parameters from the inspiral wave form?, Phys. Rev. D 49 (1994) 2658 [gr-qc/9402014] [INSPIRE].

[40] T. Damour, B.R. Iyer and B.S. Sathyaprakash, Improved filters for gravitational waves from inspiralling compact binaries, Phys. Rev. D 57 (1998) 885 [gr-qc/9708034] [INSPIRE].

[41] J.M. Fedrow et al., Gravitational Waves from Binary Black Hole Mergers inside Stars, Phys. Rev. Lett. 119 (2017) 171103 [arXiv:1704.07383] [INSPIRE].

[42] "Zero Det. High Power" curve, https://dcc.ligo.org/LIGO-T0900288/public.

[43] D.A. Brown and P.J. Zimmerman, The Effect of Eccentricity on Searches for Gravitational-Waves from Coalescing Compact Binaries in Ground-based Detectors, Phys. Rev. D 81 (2010) 024007 [arXiv:0909.0066] [INSPIRE].

[44] K. Chatziioannou, A. Klein, N. Yunes and N. Cornish, Gravitational Waveforms for Precessing, Quasicircular Compact Binaries with Multiple Scale Analysis: Small Spin Expansion, Phys. Rev. D 88 (2013) 063011 [arXiv:1307.4418] [InSPIRE].

[45] C. Capano, I. Harry, S. Privitera and A. Buonanno, Implementing a search for gravitational waves from binary black holes with nonprecessing spin, Phys. Rev. D 93 (2016) 124007 [arXiv: 1602.03509] [INSPIRE]. 